\title{
Gauge-Invariant Formulation of Fermi's Golden Rule: Application to High-Field Transport in Semiconductors
}

\author{
Emanuele Ciancio and Fausto Rossi \\ Istituto Nazionale per la Fisica della Materia (INFM) and \\ Dipartimento di Fisica, Politecnico di Torino \\ Corso Duca degli Abruzzi 24, 10129 Torino, Italy \\ FRossi@Athena.PoliTo.It
}

(November 3, 2018)

\begin{abstract}
A gauge-invariant formulation of Fermi's Golden rule is proposed. We shall rivisit the conventional description of carrier-phonon scattering in the presence of high electric fields by means of a gauge-invariant density-matrix approach. We show that the so-called intracollisional field effect - as usually accounted for-does not exist: it is simply an artifact due to the neglect of the time variation of the basis states which, in turn, leads to a ill-defined Markov limit in the carrier-phonon interaction process. This may account for the surprisingly good agreement between semiclassical and rigorous quantumtransport calculations.
\end{abstract}

72.10.-d, 72.20.Ht, 05.60.Gg

Typeset using REVTEX 
Since the early days of quantum mechanics [1] the field-induced coherent dynamics of an electron wavepacket within a crystal, known as Bloch oscillations (BO), has attracted significant and increasing interest [2]. Indeed, the problem of properly describing the scatteringfree motion of an electron in a solid has led to a three-decade controversy on the existence of BO [3]; This originated from the different approaches employed for the description of the applied field, namely the vector-potential or accelerated-Bloch-state picture [4] and the scalar-potential or Wannier-Stark description [5]. As discussed in [6], these two pictures are now recognized to be fully equivalent, since they correspond to different quantum-mechanical representations connected by a gauge transformation.

The presence of scattering as well as tunneling processes strongly modifies such ideal BO scenario [7]. In particular, non-elastic interaction mechanisms - like carrier-LO phonon scattering - tend to spoil such coherent dynamics, leading to a nearly semiclassical or Boltzmann-like transport picture. In the presence of strong electric fields, however, the use of the conventional scattering picture -involving transitions between field-free Bloch states within Fermi's golden rule- becomes questionable.

As originally pointed out by Levinson [8] and by Barker and Ferry [9], the effect of the field during the scattering process, usually referred to as intracollisional field effect (ICFE), may lead to significant deviations from the semiclassical scenario. On the one hand, the role played by the ICFE has been extensively investigated by means of rigorous quantumtransport approaches [10 13]. Their application, however, was often limited to highly simplified physical models and conditions, thus preventing from any quantitative comparison with experiments. On the other hand, strong effort has been devoted to incorporate the ICFE within conventional — and more realistic - Monte Carlo simulations [14]. In this case, the basic idea is that, due to the field-induced carrier drift, energy conservation in the scattering process is relaxed; as a consequence, the delta function of the Fermi's golden rule is replaced by broad spectral functions [15]. We stress that this scenario, intimately related to the vector-potential or accelerated picture, has no counterpart in the scalar-potential one. Indeed, within the Wannier-Stark basis there is no carrier drift, and energy conservation is 
preserved. It is thus clear that such an effective semiclassical description of the ICFE is not gauge invariant [16].

Aim of this paper is to explain and remove this apparent contraddiction by providing a rigorous - i.e., gauge-invariant - formulation of Fermi's golden rule. Our analysis will show that the ICFE, as usually accounted for within semiclassical Monte Carlo simulations [15], does not exist: it is simply an artifact due to the neglect of the time variation of the basis states which, in turn, leads to a ill-defined Markov limit in the carrier-phonon interaction process. This may account for the surprisingly good agreement between semiclassical and rigorous quantum-transport calculations [10,12].

In order to properly describe high-field transport in semiconductors, an electron-phonon system can be considered, whose Hamiltonian can be schematically written as

$$
\mathbf{H}=\mathbf{H}_{\circ}+\mathbf{H}^{\prime}=\left(\mathbf{H}_{c}+\mathbf{H}_{p}\right)+\mathbf{H}_{c p}
$$

Here, the single-particle term $\mathbf{H}_{\circ}$ includes the free-carrier and phonon Hamiltonians while the many-body contribution $\mathbf{H}^{\prime}$ accounts for carrier-phonon coupling. More specifically, the free-carrier Hamiltonian

$$
\mathbf{H}_{c}=\frac{\left(-i \hbar \nabla_{\mathbf{r}}-\frac{e}{c} \mathbf{A}(\mathbf{r}, t ; \eta)\right)^{2}}{2 m_{\circ}}+e \varphi(\mathbf{r}, t ; \eta)+V^{l}(\mathbf{r})
$$

describes the non-interacting carrier system within the periodic crystal potential $V^{l}(\mathbf{r})$ in the presence of a homogeneous external field $\mathbf{F}$, the latter being included in a fully gaugeinvariant form through the following vector and scalar potentials:

$$
\mathbf{A}(\mathbf{r}, t ; \eta)=-c \eta \mathbf{F} t, \quad \varphi(\mathbf{r} ; \eta)=(\eta-1) \mathbf{F} \cdot \mathbf{r}
$$

Here, the gauge freedom is expressed in terms of the transformation parameter $\eta$. Indeed, it is easy to verify that for any value of $\eta$ —corresponding to a different Hamiltonian $\mathbf{H}_{c}$ in Eq. (2) - we are describing exactly the same electric field F. In particular, for $\eta=0$ and $\eta=1$ one recovers the conventional scalar- and vector-potential formulations, respectively, which in turn correspond to the well-known Wannier-Stark and Bloch-oscillation pictures [6]. 
Let us finally introduce the carrier-phonon interaction Hamiltonian:

$$
\mathbf{H}_{c p}=\sum_{\mathbf{q}} \gamma_{\mathbf{q}}\left[b_{\mathbf{q}} e^{i \mathbf{q} \cdot \mathbf{r}}+b_{\mathbf{q}}^{\dagger} e^{-i \mathbf{q} \cdot \mathbf{r}}\right]
$$

where the operators $b_{\mathbf{q}}^{\dagger}\left(b_{\mathbf{q}}\right)$ describe the creation (destruction) of phonons with wavevector q.

In order to provide a gauge-invariant formulation of carrier-phonon scattering, let us consider as basis states the (gauge-dependent) eigenstates of the free-carrier Hamiltonian:

$$
\mathbf{H}_{c} \phi_{\alpha}(\mathbf{r})=\epsilon_{\alpha} \phi_{\alpha}(\mathbf{r})
$$

Here, the quantum numbers $\alpha$-and therefore the corresponding eigenfunctions $\phi_{\alpha}(\mathbf{r}) \equiv$ $\langle\mathbf{r} \mid \alpha\rangle$ and energies $\epsilon_{\alpha}$ - are functions of the transformation parameter $\eta$ and in general (i.e., for $\eta \neq 0$ ) are also functions of time. Contrary to the conventional time-dependent perturbation theory, we thus propose a kinetic description based on a time-dependent quantummechanical representation. In particular, for $\eta=0$ (scalar-potential gauge) we recover the well-known Wannier-Stark ladder [5]: $\epsilon_{\alpha}=\epsilon_{n}=\epsilon_{0}+n \Delta \epsilon$ with $\Delta \epsilon=e F d, d$ denoting the crystal periodicity along the field direction; In contrast, for $\eta=1$ we deal with the Houston or accelerated Bloch states [4]: $\epsilon_{\alpha}=\epsilon_{\mathbf{k}(t)}$, where $\mathbf{k}(t)=\mathbf{k}_{0}+\dot{\mathbf{k}} t$ is the instantaneous carrier wavevector, $\mathbf{k}=e F / \hbar$ being its field-induced time variation.

Generally speaking, we shall denote with $\mathcal{U}^{\eta, \bar{\eta}}$ the unitary transformation connecting the eigenstates $|\alpha\rangle$ in different gauges:

$$
|\alpha(\eta)\rangle=\mathcal{U}^{\eta, \bar{\eta}}|\alpha(\bar{\eta})\rangle
$$

Given such basis states $\{|\alpha\rangle\}$, most of the physical quantities we are interested in - e.g., carrier drift velocity and mean kinetic energy - are properly described by the single-particle density matrix [17]

$$
\rho_{\alpha \alpha^{\prime}}=\left\langle a_{\alpha^{\prime}}^{\dagger} a_{\alpha}\right\rangle
$$

where $a_{\alpha}^{\dagger}\left(a_{\alpha}\right)$ denote creation (destruction) operators for a carrier in state $\alpha$. This is defined as the average value of two creation and destruction operators: its diagonal elements 
$f_{\alpha}=\rho_{\alpha \alpha}$ correspond to the usual distribution functions of the semiclassical Boltzmann theory while the off-diagonal terms $\left(\alpha \neq \alpha^{\prime}\right)$ describe the degree of quantum-mechanical phase coherence between states $\alpha$ and $\alpha^{\prime}$. It is easy to show that the density matrix (7) will gauge transform according to:

$$
\rho_{\alpha_{1} \alpha_{2}}^{\eta}=\sum_{\bar{\alpha}_{3} \bar{\alpha}_{4}} U_{\bar{\alpha}_{1} \bar{\alpha}_{3}} \rho_{\bar{\alpha}_{3} \bar{\alpha}_{4}}^{\bar{\eta}} U_{\bar{\alpha}_{4} \bar{\alpha}_{2}},
$$

where $U_{\bar{\alpha} \bar{\alpha}^{\prime}}=\left\langle\alpha \mid \bar{\alpha}^{\prime}\right\rangle=\left\langle\bar{\alpha}\left|\mathcal{U}^{\bar{\eta}, \eta}\right| \bar{\alpha}^{\prime}\right\rangle$ are the matrix elements of $\mathcal{U}^{\bar{\eta}, \eta}$ in the $\bar{\eta}$ representation. Here, the compact notation $\bar{\alpha} \equiv \alpha(\bar{\eta})$ has been introduced.

For a time-dependent basis set $\{|\alpha\rangle\}$, the Heisenberg equations of motion for the operators $a_{\alpha}$ are of the form [6]:

$$
\frac{d}{d t} a_{\alpha}=\left.\frac{d}{d t} a_{\alpha}\right|_{\mathbf{H}}+\left.\frac{d}{d t} a_{\alpha}\right|_{\phi} .
$$

Compared to the standard equations of motion, the possible time variation of our basis states $\phi_{\alpha}$ gives rise to an additional term; the latter has been usually neglected, giving rise to the apparent discrepancies between scalar- and vector-potential gauges mentioned in the introductory part of the paper (see below).

By combining Eqs. (7) and (9) and considering the explicit form of the total Hamiltonian (1), we get the following equation of motion for $\rho$ :

$$
\frac{d}{d t} \rho_{\alpha \alpha^{\prime}}=-i \omega_{\alpha \alpha^{\prime}} \rho_{\alpha \alpha^{\prime}}+\left.\frac{d}{d t} \rho_{\alpha \alpha^{\prime}}\right|_{\mathbf{H}_{c p}}+\left.\frac{d}{d t} \rho_{\alpha \alpha^{\prime}}\right|_{\phi}
$$

with $\omega_{\alpha \alpha^{\prime}}=\left(\epsilon_{\alpha}-\epsilon_{\alpha^{\prime}}\right) / \hbar$. The first, Liouville-like, term is due to the single-particle Hamiltonian $\mathbf{H}_{\circ}$ while the last one is again due to the possible time variation of the basis states $\alpha$. The carrier-phonon contribution involves two-body as well as various phonon-assisted density matrices, e.g., $s_{\alpha \alpha^{\prime}, \mathbf{q}}=\left\langle a_{\alpha}^{\dagger} b_{\mathbf{q}} a_{\alpha^{\prime}}\right\rangle[17]$. These quantities describe many-particle correlations between carriers and phonons. Equation (10) is thus the starting point of an infinite hierarchy involving higher-order density matrices. To obtain a solution -i.e., a closed set of equations - this hierarchy has to be truncated at some level. As clearly discussed in [17], 
in order to properly describe carrier-phonon scattering, the time evolution of the phononassisted density matrix $s_{\alpha \alpha^{\prime}, \mathbf{q}}$ should be explicitely considered; its equation of motion has again the form of Eq. (10), i.e.,

$$
\frac{d}{d t} s_{\alpha \alpha^{\prime}, \mathbf{q}}=-i \Omega_{\alpha \alpha^{\prime}, \mathbf{q}}^{+} s_{\alpha \alpha^{\prime}, \mathbf{q}}+y_{\alpha \alpha^{\prime}, \mathbf{q}}^{c p}+\left.\frac{d}{d t} s_{\alpha \alpha^{\prime}, \mathbf{q}}\right|_{\phi}
$$

with $\Omega_{\alpha \alpha^{\prime}, \mathbf{q}}^{ \pm}=-\omega_{\alpha \alpha^{\prime}} \pm \omega_{\mathbf{q}}, \omega_{\mathbf{q}}$ being the phonon dispersion. By treating the carrier-phonon contribution $y_{\alpha \alpha^{\prime}, \mathbf{q}}^{c p}$ via the standard mean-field approximation [17, Eqs. (10) and (11) constitute a closed set of equations for the kinetic variables $\rho$ and $s$; they form the basis of the so-called carrier-phonon quantum kinetics [17].

The semiclassical limit is finally obtained via an "adiabatic elimination" [17] of the phonon-assisted density matrices $s$. This consists in a formal integration of Eq. (11) on which a Markov limit is performed. More specifically, by neglecting the $\phi$-term in Eq. (11), i.e., the contribution due to the time variation of the basis states $\alpha$, the final result is:

$$
s_{\alpha \alpha^{\prime}, \mathbf{q}}(t)=\mathcal{D}\left(\Omega_{\alpha \alpha^{\prime}, \mathbf{q}}\right) y_{\alpha \alpha^{\prime}, \mathbf{q}}^{c p}(t)
$$

with

$$
\mathcal{D}\left(\Omega_{\alpha \alpha^{\prime}, \mathbf{q}}\right)=\int_{0}^{\infty} d t e^{-i \int_{0}^{t} \Omega_{\alpha \alpha^{\prime}, \mathbf{q}}\left(t^{\prime}\right) d t^{\prime}}
$$

By inserting the above formal solution for $s$ into the carrier-phonon contribution of Eq. (10) we finally get a closed equation of motion for the single-particle density matrix $\rho$. In the linear regime, i.e., $\left|\rho_{\alpha \alpha^{\prime}}\right| \ll 1$, the carrier-phonon contribution to the dynamics is of the form:

$$
\left.\frac{d}{d t} \rho_{\alpha \alpha^{\prime}}\right|_{\mathbf{H}_{c p}}=\sum_{\beta \beta^{\prime}}\left(\tilde{\Gamma}_{\alpha \alpha^{\prime}, \beta \beta^{\prime}}^{\mathrm{in}} \rho_{\beta \beta^{\prime}}-\tilde{\Gamma}_{\alpha \alpha^{\prime}, \beta \beta^{\prime}}^{\text {out }} \rho_{\beta \beta^{\prime}}\right)+\text { c.c. }
$$

where

$$
\tilde{\Gamma}_{\alpha \alpha^{\prime}, \beta \beta^{\prime}}^{\text {in }}=\pi \sum_{ \pm, \mathbf{q}} \sum_{\alpha^{\prime \prime}} \delta_{\alpha^{\prime \prime} \beta^{\prime}} g_{\alpha \alpha^{\prime \prime}, \mathbf{q}}^{*} g_{\alpha^{\prime} \beta, \mathbf{q}} \mathcal{N}_{\mathbf{q}}^{ \pm} \mathcal{D}\left(\Omega_{\alpha^{\prime} \beta, \mathbf{q}}^{\mp}\right)
$$

and 


$$
\tilde{\Gamma}_{\alpha \alpha^{\prime}, \beta \beta^{\prime}}^{\text {out }}=\pi \sum_{ \pm, \mathbf{q}} \sum_{\alpha^{\prime \prime}} \delta_{\alpha^{\prime} \beta} g_{\alpha \alpha^{\prime \prime}, \mathbf{q}}^{*} g_{\beta^{\prime} \alpha^{\prime \prime}, \mathbf{q}} \mathcal{N}_{\mathbf{q}}^{ \pm} \mathcal{D}\left(\Omega_{\beta^{\prime} \alpha^{\prime \prime}, \mathbf{q}}^{ \pm}\right)
$$

are generalized in- and out-scattering rates [17]. Here, the \pm sign refers to phonon emission and absorption, respectively, $\mathcal{N}_{\mathbf{q}}^{ \pm}=N_{\mathbf{q}}+\frac{1}{2} \pm \frac{1}{2}$ denote the corresponding phonon occupation factors, and $g_{\alpha \beta, \mathbf{q}}$ are the matrix elements of the carrier-phonon Hamiltonian (田).

Equation (14) is the desired quantum-mechanical generalization of the well-known Boltzmann transport equation [14]; indeed, by neglecting all non-diagonal terms of the singleparticle density matrix $\left(\rho_{\alpha \alpha^{\prime}}=f_{\alpha} \delta_{\alpha \alpha^{\prime}}\right)$, the latter is easily recovered:

$$
\left.\frac{d}{d t} f_{\alpha}\right|_{H_{c p}}=\sum_{\beta}\left(\Gamma_{\alpha \beta} f_{\beta}-\Gamma_{\beta \alpha} f_{\alpha}\right) .
$$

Here, as usual, the scattering rates for in- and out-scattering processes coincide; they correspond to twice the diagonal parts $\left(\alpha \beta=\alpha^{\prime} \beta^{\prime}\right)$ of the scattering operators $\tilde{\Gamma}^{\text {in }}$ and $\tilde{\Gamma}^{\text {out }}$ in Eq. (15):

$$
\Gamma_{\alpha \beta}=\Gamma_{\beta \alpha}=2 \pi \sum_{ \pm, \mathbf{q}}\left|g_{\alpha \beta, \mathbf{q}}\right|^{2} \mathcal{N}_{\mathbf{q}}^{ \pm} \Re\left[\mathcal{D}\left(\Omega_{\alpha \beta, \mathbf{q}}^{ \pm}\right)\right]
$$

The above semiclassical rates exhibit the well-known structure of the Fermi's golden rule; they describe the scattering probability for a phonon-induced transition between states $\alpha$ and $\beta$. Their quantum-mechanical - or non-diagonal - generalization is then given by the scattering matrices (15), which describe the effect on the time evolution of the density-matrix element $\rho_{\alpha \alpha^{\prime}}$ due to the generic element $\rho_{\beta \beta^{\prime}}$.

The generalized carrier-phonon scattering rates in (15) — as well as their semiclassical counterparts in $(\sqrt{17})$ - involve the $\mathcal{D}$ function in $(\sqrt{13})$. For the case of a time-independent basis set, i.e., $\eta=0$ (Wannier-Stark states), the detuning frequency $\Omega$ is also time-independent and the real part of the function $\mathcal{D}$ in (13) gives the well-known energy-conserving Dirac delta function of Fermi's golden rule; in contrast, for the case of a time-dependent basis, i.e., $\eta=1$ (accelerated Bloch states), the detuning is time-dependent, leading to a function $\mathcal{D}$ with a Fresnel-like shape [10]. This is exactly the ICFE previously introduced [9]: due to the field-induced variation of the carrier wavevector $\mathbf{k}$, the energy difference between initial and final states $\left(\epsilon_{\mathbf{k}(t)}-\epsilon_{\mathbf{k}(t) \pm \mathbf{q}}\right)$ changes in time giving rise to broad resonances in the 
carrier-phonon scattering process. Such energy-nonconserving scenario has no counterpart in the Wannier-Stark picture [18]. This clearly shows that the generalized scattering rates in (15) are not gauge invariant.

Aim of this paper is to show that (i) the derivation recalled so far is only valid within the Wannier-Stark picture $(\eta=0)$ and (ii) the ICFE previously described is simply an artifact due to the approximation scheme usually considered. Indeed, as anticipated, the crucial point is the neglect of the $\phi$-terms, i.e., of the possible time variation of our basis states $\alpha$. More specifically, a proper inclusion of these terms leads to a modified version of Eq. (11):

$$
\frac{d}{d t} s_{\alpha \alpha^{\prime}, \mathbf{q}}=i \sum_{\beta \beta^{\prime}} \tilde{\Omega}_{\alpha \alpha^{\prime}, \beta \beta^{\prime}, \mathbf{q}}^{+} s_{\beta \beta^{\prime}, \mathbf{q}}+y_{\alpha \alpha^{\prime}, \mathbf{q}}^{c p}
$$

with

$$
\hbar \tilde{\Omega}_{\alpha \alpha^{\prime}, \beta \beta^{\prime}, \mathbf{q}}^{ \pm}=\mathcal{E}_{\alpha \beta} \delta_{\alpha^{\prime} \beta^{\prime}}-\mathcal{E}_{\alpha^{\prime} \beta^{\prime}} \delta_{\alpha \beta} \pm \hbar \omega_{\mathbf{q}} \delta_{\alpha \beta} \delta_{\alpha^{\prime} \beta^{\prime}}
$$

$\mathcal{E}_{\alpha \alpha^{\prime}}$ being the matrix elements of the single-particle Hamiltonian (2) for $\eta=0$, i.e., written in the scalar-potential gauge. It follows that for a generic time-dependent basis, Eq. (18) has a non-diagonal structure,i.e., it does not allow a simple exponential solution. This implies that for $\eta \neq 0$ the Markov limit is not straightforward. Indeed, the rigorous procedure is: (i) to perform a unitary transformation which diagonalizes the superoperator $\tilde{\Omega}$ in (19), and (ii) to perform the exponential formal integration described above. Since $\mathcal{E}_{\alpha \alpha^{\prime}}$ are the matrix elements of $\mathbf{H}_{c}$ for $\eta=0$ (scalar-potential gauge), the unitary transformation that diagonalizes $\tilde{\Omega}$ is just $\mathcal{U}^{0, \eta}$, i.e., the transformation connecting the generic gauge $\eta$ to the scalar-potential basis $(\eta=0)$. We stress that the new diagonal elements coincide with the eigenvalues of $\tilde{\Omega}$ which, in turn, correspond to the time-independent detuning functions $\Omega_{\alpha \alpha^{\prime}, \mathbf{q}}$ in the Wannier-Stark gauge. This clearly shows that the Markov limit used to derive the generalized Boltzmann equation in (14) is only well-defined in the Wannier-Stark picture, for which the various $\phi$-terms vanish and the detuning functions $\Omega$ are time-independent. This does not violate the gauge-invariant nature of our formulation. Indeed, given the generalized Boltzmann equation (14) written in the scalar-potential picture, the latter can be written 
in any generic gauge $\eta$ by applying the unitary transformation $\mathcal{U}$ introduced in (6). More specifically, let us introduce the single-particle density-matrix operator

$$
\rho=\sum_{\alpha \alpha^{\prime}}|\alpha\rangle \rho_{\alpha \alpha^{\prime}}\left\langle\alpha^{\prime}\right|
$$

which is by definition gauge invariant, i.e., $\eta$-independent. This can be easily checked by combining Eqs. (6) and (8). This suggests to write the generalized Boltzmann equation (14) in an operatorial form as:

$$
\left.\frac{d}{d t} \rho\right|_{\mathbf{H}_{c p}}=\left(\tilde{\Gamma}^{\text {in }} \rho-\tilde{\Gamma}^{\text {out }} \rho\right)+\text { h.c. }
$$

where

$$
\tilde{\Gamma}^{\text {in/out }}=\sum_{\alpha \alpha^{\prime}, \beta \beta^{\prime}}|\alpha\rangle|\beta\rangle \tilde{\Gamma}_{\alpha \alpha^{\prime}, \beta \beta^{\prime}}^{\text {in } / \text { out }}\left\langle\alpha^{\prime}\right|\left\langle\beta^{\prime}\right|
$$

are in- and out-scattering superoperators. Our aim is to propose a gauge-invariant formulation of the problem. This requires the superoperators in (22) to be $\eta$-independent as well. The analysis presented so far has shown that the scattering matrices $\tilde{\Gamma}_{\alpha \alpha^{\prime}, \beta \beta^{\prime}}^{\text {in/out }}$ are only well defined in the Wannier-Stark picture $(\eta=0)$. This allows us to extend their definitions to the generic $\eta$ representation according to:

$$
\tilde{\Gamma}_{\alpha_{1} \alpha_{2}, \beta_{1} \beta_{2}}^{\eta}=U_{\bar{\alpha}_{1} \bar{\alpha}_{3}} U_{\bar{\beta}_{1} \bar{\beta}_{3}} \tilde{\Gamma}_{\bar{\alpha}_{3} \bar{\alpha}_{4}, \bar{\beta}_{3} \bar{\beta}_{4}}^{\bar{\eta}=0} U_{\bar{\alpha}_{4} \bar{\alpha}_{2}} U_{\bar{\beta}_{4} \bar{\beta}_{2}}
$$

where $U_{\bar{\alpha}} \bar{\alpha}^{\prime}$ are the matrix elements of the gauge transformation $\mathcal{U}^{0, \eta}$ in the Wannier-Stark picture $(\bar{\eta}=0)$. Here, implicit summation over repeated indices is assumed.

Equation (23) is the gauge-invariant formulation of Fermi's golden rule we were looking for. Contrary to the conventional approach, in the case of a time-dependent basis, e.g., accelerated Bloch states [4], instead of using Eq. (15) with an ad hoc energy-nonconserving $\mathcal{D}$ function, the rigorous procedure is to compute the generalized scattering rates (15) in the Wannier-Stark picture, and then to apply the gauge transformation $\mathcal{U}_{\eta, 0}$ according to Eq. (23).

In summary, we have proposed a gauge-invariant formulation of carrier-phonon interaction. It has been shown that ICFE — as usually described and accounted for - is just an 
artefact due to the neglect of the time variation of the basis set. As anticipated, this may account for the surprisingly good agreement between semiclassical and rigorous quantumtransport calculations reported in [10] and [12], as well as for the anomalous carrier heating typical of semiclassical ICFE models [15].

From our analysis we can conclude that the most severe approximation of the Boltzmann transport theory is not the Markov limit but the semiclassical approximation, i.e., the neglect of non-diagonal density-matrix elements. The latter, being intrinsically basis dependent, is not compatible with a gauge-invariant formulation of the problem. 


\section{REFERENCES}

[1] F. Bloch, Z. Phys. 52, 555 (1928).

[2] see, e.g., J. Shah, Ultrafast Spectroscopy of Semiconductors and Semiconductor Nanostructures (Springer, Berlin, 1996); Theory of Transport Properties of Semiconductor Nanostructures, edited by E. Schöll (Chapman \& Hall, London 1998).

[3] See, e.g., G. Nenciu, Rev. Mod. Phys. 63, 91 (1991).

[4] W.V. Houston, Phys. Rev. 57, 184 (1940).

[5] G.H. Wannier, Rev. Mod. Phys. 34, 645 (1962).

[6] F. Rossi, in Theory of Transport Properties of Semiconductor Nanostructures, edited by E. Schöll (Chapman \& Hall, London 1998), p. 283.

[7] See, e.g., T. Meier, F. Rossi, P. Thomas, and S.W. Koch, Phys. Rev. Lett. 75, 2558 (1995); F. Rossi, A. Di Carlo, and P. Lugli, Phys. Rev. Lett. 80, 3348 (1998); B. Rosam et al., Phys. Rev. Lett. 86, 1307 (2001).

[8] I.B. Levinson and Y. Yasevichyute, Sov. Phys. JETP 35, 991 (1972).

[9] J.F. Barker and D.K. Ferry, Phys. Rev. Lett. 42, 1779 (1979).

[10] R. Brunetti, C. Jacoboni, and F. Rossi, Phys. Rev.B 39, 10781 (1989).

[11] R. Bertoncini and A.P. Jauho, Phys. Rev.B 44, 3655 (1991).

[12] M. Herbst, V.M. Axt, T. Kuhn, and J. Schilp, phys. stat. sol. (b) 204, 358 (1997).

[13] J. Hader et al., Phys. Rev.B 55, 13799 (1997).

[14] See, e.g., C. Jacoboni and P. Lugli, The Monte Carlo Method for Semiconductor Device Simulations (Springer, Wien 1989).

[15] See, e.g., L. Reggiani, P. Lugli, and A.P. Jauho, Phys. Rev.B 36, 6602 (1987) and references therein. 
[16] The need for a gauge-invariant formulation of the problem was originally pointed out by Bertoncini and Jauho in [11].

[17] T. Kuhn, in Theory of Transport Properties of Semiconductor Nanostructures, edited by E. Schöll (Chapman \& Hall, London 1998), p. 173.

[18] In addition to "energy-nonconserving effects" due to a time-dependent basis, we also deal with energy-broadening effects, the socalled "collisional broadening" [11. Such effects can be easily included in our theoretical approach by adding an imaginary contribution to the self-energy $\Omega$ in $(13)$. 